\title{
Towards Non-Commutative Deformations of Relativistic Wave Equations in 2+1 Dimensions ${ }^{\star}$
}

\author{
Bernd J. SCHROERS ${ }^{\dagger}$ and Matthias WILHELM ${ }^{\ddagger}$ \\ $\dagger$ Department of Mathematics and Maxwell Institute for Mathematical Sciences, \\ Heriot-Watt University, Edinburgh EH14 4AS, UK \\ E-mail: b.j.schroers@hw.ac.uk \\ URL: http://www.macs.hw.ac.uk/ bernd/ \\ ‡ Institut für Mathematik und Institut für Physik, Humboldt-Universität zu Berlin, \\ IRIS-Adlershof, Zum Großen Windkanal 6, 12489 Berlin, Germany \\ E-mail: mwilhelm@physik.hu-berlin.de
}

Received February 28, 2014, in final form May 09, 2014; Published online May 20, 2014

http://dx.doi.org/10.3842/SIGMA.2014.053

\begin{abstract}
We consider the deformation of the Poincaré group in $2+1$ dimensions into the quantum double of the Lorentz group and construct Lorentz-covariant momentum-space formulations of the irreducible representations describing massive particles with spin $0, \frac{1}{2}$ and 1 in the deformed theory. We discuss ways of obtaining non-commutative versions of relativistic wave equations like the Klein-Gordon, Dirac and Proca equations in $2+1$ dimensions by applying a suitably defined Fourier transform, and point out the relation between non-commutative Dirac equations and the exponentiated Dirac operator considered by Atiyah and Moore.
\end{abstract}

Key words: relativistic wave equations; quantum groups; curved momentum space; noncommutative spacetime

2010 Mathematics Subject Classification: 83A99; 81R20; 81R50; 81R60

\section{Introduction}

It is well-known that the important linear wave equations of relativistic physics can be obtained by Fourier transforming the irreducible representations of the Poincaré group. The KleinGordon, Dirac and Proca equations, for example, are Fourier transforms of momentum-space constraints for, respectively, spin $0, \frac{1}{2}$ and 1 in Wigner's classification of irreducible Poincaré representations in terms of mass and spin [8, 43].

In this paper, we discuss this picture for the case of $(2+1)$-dimensional Minkowski space, and then consider a deformation of it where the Poincaré symmetry is deformed into a noncocommutative quantum group, namely the quantum double of the Lorentz group in $2+1$ dimensions, or Lorentz double for short $[6,7,26]$. The deformation involves a parameter of dimension inverse mass, and deforms flat momentum space of ordinary special relativity into anti-de Sitter space; in $2+1$ dimensions, this happens to be isometric to the identity component of the Lorentz group.

The Lorentz double plays an important role in $(2+1)$-dimensional quantum gravity [6, 7, 33, $34,40]$. In that context, the deformation parameter is related to Newton's constant. We will not discuss the gravitational interpretation much in this paper and refer to the review [41] for details and references. Instead we focus on general, structural features of our $(2+1)$-dimensional

${ }^{\star}$ This paper is a contribution to the Special Issue on Deformations of Space-Time and its Symmetries. The full collection is available at http://www.emis.de/journals/SIGMA/space-time.html 
example, treating it as a case study of more general deformations of momentum spaces to curved manifolds.

Such deformations have been considered in various guises and with different motivations in the physics literature. Early considerations of curvature in momentum space include the work of Born [11] on a duality between position and momentum, and also the influential paper by Snyder [42] where momentum space is taken to be the de Sitter manifold. Majid's bicrossproduct construction $[28,29]$ provides a mathematical framework for deforming spacetime symmetries which naturally accommodates curved momentum space. The famous deformation of Poincaré symmetry into the $\kappa$-Poincaré algebra [27] was later seen to fit into this framework [30]. In recent years, phenomenological implications of these ideas have been explored extensively under the headings of 'doubly special relativity' [2] and 'relative locality' [3].

In our $(2+1)$-dimensional theory, position coordinates, which are translation generators in momentum space, no longer commute. Instead, they satisfy the Lie algebra of the Lorentz group in $2+1$ dimensions and act on the Lorentz group-valued momenta by infinitesimal multiplication (see [32] for an early discussion of this point and [41] for a review and further references). One therefore expects that Fourier transforming the irreducible representations of the Lorentz double, where states are functions on momentum spaces, will lead to covariant wave equations on a noncommutative spacetime.

In this paper, we take the first steps towards realising that expectation. Our treatment follows a similar discussion of the Euclidean situation in [31], which is our main reference. As we shall see, the Lorentzian situation is considerably more involved than the Euclidean case.

We begin, in Section 2, by writing the unitary irreducible representations (UIR's) of the usual $(2+1)$-dimensional Poinaré group in a covariant form that allows us to obtain relativistic wave equations via Fourier transform. Even though the wave equations we obtain are the standard Klein-Gordon, Dirac and Proca equation in $2+1$ dimensions, our method for obtaining them does not appear to have been considered in the literature.

A full classification the UIR's of the Poincaré group in $2+1$ dimensions was first given by Binegar in [10], where he also discusses the possibility - and difficulties - of writing the UIR's in terms of fields on Minkowski space obeying covariant wave equations. A complete analysis of relativistic wave equations in $2+1$ dimensions is given in [18] from the point of view of generalised regular representations. Our approach gives a less general treatment of the Poincaré UIR's, but maintains the link via Fourier transform between momentum space and position space. This link is essential in our derivation of non-commutative wave equations from irreducible representations of the Lorentz double in subsequent sections.

In Section 3, we review the representation theory of the Lorentz double and then adapt the covariantisation procedure developed in Section 2 to the irreducible representations of the Lorentz double, still following the treatment of the Euclidean situation in [31]. Section 4 is concerned with the translation of the momentum space constraints into a spacetime picture. We use two different kinds of Fourier transform to obtain wave equations from the covariant momentum constraints. One is a Fourier transform adapted to quantum groups [24, 29] where 'plane waves' are elements of the Lorentz group and the resulting wave equations are defined on the (suitably completed) universal enveloping algebra of the Lie algebra of the Lorentz group. The second is a group Fourier transform which, in the model considered here, leads to wave equations for functions on $\mathbb{R}^{3}$ with a certain $\star$-product $[15,16,17,20,21,38,39]$. We discuss the relationship between the various notions of Fourier transform and point out an interesting connection with the exponentiated Dirac operator recently proposed by Atiyah and Moore in [5].

As a caveat we should say that our treatment in Section 4 is far from complete; it is designed to point out interesting questions posed by the results of our Section 3 and to prepare the ground for tackling them. Examples of such questions are discussed in our final Section 5, which contains our conclusion and outlook. 


\section{Relativistic wave equations in $2+1$ dimensions}

\subsection{Conventions and notation}

We denote $(2+1)$-dimensional Minkowski space by $\mathbb{R}^{2,1}$ and use the 'mostly minus' convention for the Minkowski metric $\eta=\operatorname{diag}(1,-1,-1)$. We write elements of $\mathbb{R}^{2,1}$ as $x, y, \ldots$ with $x=\left(x^{0}, x^{1}, x^{2}\right)$ and

$$
\eta(x, y)=\eta_{a b} x^{a} y^{b}=x^{0} y^{0}-x^{1} y^{1}-x^{2} y^{2} .
$$

Latin indices range over $0,1,2$ and summation over repeated indices is implied.

The group of linear transformations of $\mathbb{R}^{2,1}$ that leave $\eta$ invariant is the Lorentz group $L_{3}=$ $\mathrm{O}(2,1)$. It has four connected components. We are mainly interested in the identity component the subgroup of proper orthochronous Lorentz transformations, denoted $L_{3}^{+\uparrow}$.

The group of affine transformations that leave the Minkowski distance $\eta(x-y, x-y)$ invariant is the semidirect product $L_{3} \ltimes \mathbb{R}^{3}$ of the Lorentz group with the abelian group of translations. We call it the extended Poincaré group. Its identity component is the Poincaré group, which we denote as

$$
P_{3}=L_{3}^{+\uparrow} \ltimes \mathbb{R}^{3} .
$$

For the semidirect product we use the conventions of [31], which allow for an easy extension to the quantum group deformation in the next section but are different from those mostly used in the physics literature. In our conventions, the product of $\left(\Lambda_{1}, a_{1}\right),\left(\Lambda_{2}, a_{2}\right) \in P_{3}$ is given by

$$
\left(\Lambda_{1}, a_{1}\right)\left(\Lambda_{2}, a_{2}\right)=\left(\Lambda_{1} \Lambda_{2}, \Lambda_{2} a_{1}+a_{2}\right) .
$$

One advantage of this convention is that the ordering of the elements can be interpreted as a factorisation: $(\Lambda, a)=(\Lambda, 0)(\mathrm{I}, a)$, where $\mathrm{I}$ is the identity in $\mathrm{O}(2,1)$.

The action of $(\Lambda, a) \in P_{3}$ on the Minkowski space is then the right action

$$
(\Lambda, a): x \mapsto x \triangleleft(\Lambda, a)=\Lambda x+a .
$$

For a full classification of possible excitations in $(2+1)$-dimensional relativistic physics, including the anyonic ones, one needs to study the projective UIR's of $P_{3}$. These are given by the ordinary UIR's of the universal covering group of $P_{3}$, which are studied in detail in [19]. Wave equations for anyonic wave functions with infinitely many components are investigated in [22]. In this paper, we work with the double cover $\operatorname{SL}(2, \mathbb{R})$ of $L_{3}^{+\uparrow}$ and hence the double cover $\tilde{P}_{3}=\mathrm{SL}(2, \mathbb{R}) \ltimes \mathbb{R}^{3}$ of the Poincaré group. The main reason for this is the convenience of working with $2 \times 2$ matrices, and an easier link with the existing literature on the Lorentz double, which mostly uses a formulation based on $\operatorname{SL}(2, \mathbb{R})$. Note also that, in $3+1$ dimensions, the double cover of the Poincaré group is the universal cover.

It turns out to be natural and convenient to interpret the translation group $\mathbb{R}^{3}$ as the vector space $\mathfrak{s l}(2, \mathbb{R})^{*}$ dual to $\mathfrak{s l}(2, \mathbb{R})$. Then $\tilde{P}_{3}=\operatorname{SL}(2, \mathbb{R}) \ltimes \mathfrak{s l}(2, \mathbb{R})^{*}$, where $\operatorname{SL}(2, \mathbb{R})$ acts on $\mathfrak{s l}(2, \mathbb{R})^{*}$ via the coadjoint action. The right-action of $(g, a) \in \tilde{P}_{3}$ on Minkowski space $\mathfrak{s l}(2, \mathbb{R})^{*}$ is then given by

$$
(g, a): \mathfrak{s l}(2, \mathbb{R})^{*} \ni x \mapsto x \triangleleft(g, a)=\operatorname{Ad}_{g}^{*} x+a .
$$

This action preserves the Minkowski metric $\eta$ on $\mathfrak{s l}(2, \mathbb{R})^{*}{ }^{1}$

\footnotetext{
${ }^{1}$ We can think of $\eta$ as being induced by the Killing form on the dual $\left(\mathfrak{s l}(2, \mathbb{R})^{*}\right)^{*}$, but this is not essential in the following.
} 
The Lie algebra $\mathfrak{p}_{3}=\mathfrak{s l}(2, \mathbb{R}) \ltimes \mathfrak{s l}(2, \mathbb{R})^{*}$ is six dimensional, with translation generators $P_{0}, P_{1}$ and $P_{2}$, rotation generator $J_{0}$ and boost generators $J_{1}$ and $J_{2}$. They satisfy the commutation relations:

$$
\left[J_{a}, J_{b}\right]=\epsilon_{a b c} J^{c}, \quad\left[J_{a}, P_{b}\right]=\epsilon_{a b c} P^{c}, \quad\left[P_{a}, P_{b}\right]=0, \quad a, b=0,1,2
$$

where indices are raised via the inverse Minkowski metric $\eta^{a b}$ and $\epsilon_{a b c}$ is the totally antisymmetric tensor in three dimensions normalised such that $\epsilon_{012}=1$. We are using conventions where the structure constants in the Lie algebra are real. This has the advantage that we can exponentiate to obtain group elements without needing to insert the imaginary unit $i$. Our conventions differ from those in [31] in this respect.

The vector spaces $\mathfrak{s l}(2, \mathbb{R})$ and $\mathfrak{s l}(2, \mathbb{R})^{*}$, which make up $\mathfrak{p}_{3}$, are in duality, and the natural pairing between them is invariant and non-degenerate. This pairing plays an important role in the Chern-Simons formulation of $2+1$ gravity [1, 41, 45], where it is normalised via Newton's constant $G$ :

$$
\left\langle J_{a}, P_{b}\right\rangle=\frac{1}{8 \pi G} \eta_{a b}, \quad\left\langle J_{a}, J_{b}\right\rangle=\left\langle P_{a}, P_{b}\right\rangle=0
$$

\subsection{Irreducible unitary representations of $\tilde{P}_{3}$}

The UIR's of $\tilde{P}_{3}$ are classified in terms of $\operatorname{SL}(2, \mathbb{R})$ orbits in $\left(\mathfrak{s l}(2, \mathbb{R})^{*}\right)^{*}$ together with UIR's of associated stabiliser groups [8]. Since $\left(\mathfrak{s l}(2, \mathbb{R})^{*}\right)^{*}=\mathfrak{s l}(2, \mathbb{R})$, these orbits are nothing but adjoint orbits of $\operatorname{SL}(2, \mathbb{R})$. The following is a convenient basis of $\mathfrak{s l}(2, \mathbb{R})$, whose detailed properties are summarised in Appendix A:

$$
t^{0}=\frac{1}{2}\left(\begin{array}{rr}
0 & 1 \\
-1 & 0
\end{array}\right), \quad t^{1}=\frac{1}{2}\left(\begin{array}{rr}
1 & 0 \\
0 & -1
\end{array}\right), \quad t^{2}=\frac{1}{2}\left(\begin{array}{ll}
0 & 1 \\
1 & 0
\end{array}\right) .
$$

However, we need to be careful about normalisation. The normalisation of $\left\{t^{a}\right\}_{a=0,1,2}$ is fixed by the commutation relations (A.3). The normalisation of the basis $\left\{P^{* a}\right\}_{a=0,1,2}$, which is dual to the basis $\left\{P_{a}\right\}_{a=0,1,2}$ used in (2.1), may be different. Therefore, we should allow

$$
P^{* a}=\lambda t^{a}, \quad a=0,1,2,
$$

where $\lambda$ is an arbitrary constant of dimension inverse mass. In Section 3, we use the invariant pairing $(2.2)$ to identify $\mathfrak{s l}(2, \mathbb{R})^{*}$ with $\mathfrak{s l}(2, \mathbb{R})$, and $P^{* a}$ with $8 \pi G J^{a}$. The commutation relations (2.1) then fix $\lambda=8 \pi G$.

We denote elements of momentum space $\mathfrak{s l}(2, \mathbb{R})$ as $p$, which we expand as

$$
p=p_{a} P^{* a}=\lambda p_{a} t^{a} .
$$

The adjoint action of $\mathrm{SL}(2, \mathbb{R})$ on $\mathfrak{s l}(2, \mathbb{R})$ leaves invariant the inner product

$$
-\frac{2}{\lambda^{2}} \operatorname{tr}(p q)=p_{a} q^{a}
$$

In the following, we take $p^{2}$ to mean $p_{a} p^{a}$, not the square of the matrix $p$.

The orbits of the $\operatorname{SL}(2, \mathbb{R})$ adjoint action on $p \in \mathfrak{s l}(2, \mathbb{R})$ are labelled by the value of the invariant inner product $p^{2}$. The different cases are naturally distinguished by the timelike $(\mathrm{T})$, spacelike (S) or lightlike (L) nature of the elements $p$ on a given orbit.

T: There are two disjoint families of orbits, corresponding to the different possible signs of a real parameter $m \neq 0$. Starting from the timelike representative element $\hat{p}=\lambda m t^{0}$, the orbits

$$
O_{m}^{T}=\left\{v \lambda m t^{0} v^{-1} \mid v \in \mathrm{SL}(2, \mathbb{R})\right\}=\left\{\lambda p_{a} t^{a} \in \mathfrak{s l}(2, \mathbb{R}) \mid p^{2}=m^{2}, \frac{p_{0}}{m}>0\right\}
$$


are the 'forward' and 'backward' sheets of the two-sheeted mass hyperboloid for, respectively, $m>0$ and $m<0$. The associated stabiliser group is

$$
N^{T}=\left\{\exp \left(\phi t^{0}\right) \mid \phi \in[0,4 \pi)\right\} \simeq \mathrm{U}(1) .
$$

Its UIR's are labelled by $s \in \frac{1}{2} \mathbb{Z}$; the half-integer values arise because of the range of $\phi$ for elements of the form $e^{\phi t^{0}} \in \mathrm{SL}(2, \mathbb{R})$.

The parameters $|m|$ and $s$ can be interpreted as the mass and the spin of a particle. We allow $m$ to be either positive or negative, corresponding to the cases of a particle or antiparticle. Further note that, in contrast to the $3+1$ dimensional case, the spin $s$ can also be either positive or negative. In fact, spin in $2+1$ dimensions violates parity $P$ and time-reversal $T$ unless two species with opposite spin are included in a theory [12].

S: Picking a typical spacelike representative element $\hat{p}=\lambda \mu t^{1}$, the resulting orbit

$$
O_{\mu}^{S}=\left\{v \lambda \mu t^{1} v^{-1} \mid v \in \mathrm{SL}(2, \mathbb{R})\right\}=\left\{\lambda p_{a} t^{a} \in \mathfrak{s l}(2, \mathbb{R}) \mid p^{2}=-\mu^{2}<0\right\}
$$

is a single-sheeted hyperboloid. The real parameter $\mu$ is strictly positive. The associated stabiliser is

$$
N^{S}=\left\{ \pm \exp \left(\vartheta t^{1}\right) \mid \vartheta \in \mathbb{R}\right\} \simeq \mathbb{R} \times \mathbb{Z}_{2},
$$

and its UIR's are labelled by pairs $(s, \epsilon)$, with $s \in \mathbb{R}, \epsilon= \pm 1$. Empirically, particles with spacelike momenta - so-called tachyons - do not exist in the physical $3+1$ dimensions.

L: There are again two possibilities corresponding to the different possible signs of $p_{0}$. Picking the lightlike representative elements $\hat{p}= \pm E^{+}= \pm\left(t^{0}+t^{2}\right)$ introduced in (A.5), we obtain the 'forward' and 'backward' light cones as orbits:

$$
O^{L \pm}=\left\{ \pm v E^{+} v^{-1} \mid v \in \mathrm{SL}(2, \mathbb{R})\right\}=\left\{\lambda p_{a} t^{a} \in \mathfrak{s l}(2, \mathbb{R}) \mid p^{2}=0, \pm p_{0}>0\right\} .
$$

The stabiliser group in both cases is

$$
N^{L}=\left\{ \pm \exp \left(z E^{+}\right) \mid z \in \mathbb{R}\right\} \simeq \mathbb{R} \times \mathbb{Z}_{2} .
$$

Its UIR's are again labelled by pairs $(s, \epsilon)$, with $s \in \mathbb{R}, \epsilon= \pm 1$.

V: The 'vacuum' orbit $\{0\}$ consists solely of the origin and the associated stabiliser is the whole group $\operatorname{SL}(2, \mathbb{R})$. The irreducible representations of $\operatorname{SL}(2, \mathbb{R})$ can, for instance, be found in $[25]$.

There are two standard ways of writing down the UIR's of semidirect product groups like $\tilde{P}_{3}$, both using the orbits and stabiliser UIR's listed above. One uses sections of bundles over the homogeneous space $\mathrm{SL}(2, \mathbb{R}) / N$, where $N$ denotes one of the stabiliser groups. The group action on such sections involves multipliers or cocycles, see [8] for details. The other uses functions on $\operatorname{SL}(2, \mathbb{R})$ satisfying an equivariance condition. This is the formulation we use here, referring the reader to $[7,8]$ for a translation between the two approaches.

For a given UIR of $\tilde{P}_{3}$ labelled by an orbit $O$ with representative element $\hat{p}$, stabiliser group $N$ and UIR $\varsigma$ of $N$ on a vector space $V$, the carrier space is

$$
V_{O, \varsigma}=\left\{\psi: \operatorname{SL}(2, \mathbb{R}) \rightarrow V \mid \psi(v n)=\varsigma\left(n^{-1}\right) \psi(v), \forall n \in N, \forall v \in \operatorname{SL}(2, \mathbb{R})\right\} .
$$

We also have to impose an integrability condition, which we give in a particular case below. An element $(g, a) \in \tilde{P}_{3}$ acts on $\psi \in V_{O, \varsigma}$ via

$$
\pi_{O, \varsigma}((g, a)) \psi(v)=\exp \left(i a\left(\operatorname{Ad}_{g^{-1} v}(\hat{p})\right)\right) \psi\left(g^{-1} v\right) .
$$


As we will subsequently focus on the case of timelike momenta, we give the carrier space for this case explicitly:

$$
V_{m s}=\left\{\psi: \mathrm{SL}(2, \mathbb{R}) \rightarrow \mathbb{C} \mid \psi\left(v e^{\alpha t^{0}}\right)=e^{-i s \alpha} \psi(v), \forall(\alpha, v) \in[0,4 \pi) \times \mathrm{SL}(2, \mathbb{R})\right\} .
$$

The integrability condition is

$$
\int_{\mathrm{SL}(2, \mathbb{R}) / N^{T}}|\psi|^{2}(w) d \nu(w)<\infty
$$

Here, $d \nu$ is the invariant measure on the homogeneous space $\operatorname{SL}(2, \mathbb{R}) / N^{T}$ (note that $|\psi|^{2}$ only depends on $\left.w \in \mathrm{SL}(2, \mathbb{R}) / N^{T}\right)$.

An element $(g, a) \in \tilde{P}_{3}$ acts on $\psi \in V_{m s}$ via

$$
\pi_{m s}((g, a)) \psi(v)=\exp \left(i a\left(\operatorname{Ad}_{g^{-1} v}\left(\lambda m t^{0}\right)\right)\right) \psi\left(g^{-1} v\right) .
$$

If we introduce the notation

$$
p=\lambda m v t^{0} v^{-1}
$$

for an orbit element, this further simplifies to

$$
\pi_{m s}((g, a)) \psi(v)=e^{i a\left(\operatorname{Ad}_{g^{-1}}(p)\right)} \psi\left(g^{-1} v\right) .
$$

\subsection{Covariant momentum constraints}

In a field theory, we are usually looking for wave functions that are defined on momentum or position space and which transform covariantly under the action of the Poincaré group [8, 10]. In our conventions, the required transformation behaviour reads

$$
\pi((g, a)) \tilde{\phi}(p)=e^{i a\left(\operatorname{Ad}_{g^{-1}}(p)\right)} \rho(g) \tilde{\phi}\left(g^{-1} p\right),
$$

where $\rho$ is a (preferably finite-dimensional) representation of the full group $\operatorname{SL}(2, \mathbb{R})$.

To obtain a covariant description, we employ the technique of [31]. In geometric terms, the approach taken there can be described as follows. The formulation (2.5) defines elements of the carrier space of an UIR as functions on the group obeying an equivariance condition. Replacing $\mathrm{SL}(2, \mathbb{R})$ with a general Lie group $G$ and considering a general stabiliser subgroup $N$, this is nothing but the equivariant description of sections of vector bundles over $G / N$. For $G=\mathrm{SU}(2)$ and $N=\mathrm{U}(1)$, these are the standard Hermitian line bundles over $S^{2}$.

The trick used in [31] is to view the bundles above as subbundles of the trivial bundle $S^{2} \times \mathbb{C}^{n}$, where $\mathbb{C}^{n}$ is the standard $n$-dimensional UIR of $\mathrm{SU}(2)$. In that way, sections become ordinary functions $S^{2} \rightarrow \mathbb{C}^{n}$ obeying a linear constraint. In this construction, the unitarity of the $\mathrm{SU}(2)$ action on $\mathbb{C}^{n}$ is essential for obtaining Hermitian line bundles. By thinking of $S^{2}$ as embedded in Euclidean (momentum) 3-space, one arrives at functions $\mathbb{R}^{3} \rightarrow \mathbb{C}^{n}$ obeying linear constraints. Applying an ordinary Fourier transform then produces functions on Euclidean (position) 3-space obeying a linear differential equation.

We would like to treat the Lorentzian situation analogously. However, the standard $n$ dimensional irreducible representations of $\operatorname{SL}(2, \mathbb{R})$, reviewed in Appendix A, are not unitary, and therefore the procedure of [31] cannot be used to obtain all UIR's of $\tilde{P}_{3}$. We shall now show that it can be implemented for the UIR's (2.6) labelled by orbits containing timelike momenta. In that case, the stabiliser group is the $\mathrm{U}(1)$ subgroup of $\mathrm{SL}(2, \mathbb{R})$ generated by $t^{0}$.

For a given $\psi$ in (2.6), we define the maps

$$
\tilde{\phi}: O_{m}^{T} \rightarrow \mathbb{C}^{2|s|+1}
$$


via

$$
\tilde{\phi}(p)=\psi(v) \rho^{|s|}(v)|| s|, s\rangle,
$$

where $p$ is related to $v$ via (2.7), and the states ||$s|, k\rangle$ form the basis (A.6) of the finitedimensional $\mathfrak{s l}(2, \mathbb{R})$ irreducible representations in which $t^{0}$ is diagonal. Clearly,

$$
\rho^{|s|}\left(v e^{\alpha t^{0}}\right)|| s|, s\rangle=\rho^{|s|}(v) \rho^{|s|}\left(e^{\alpha t^{0}}\right)|| s|, s\rangle=\rho^{|s|}(v) e^{i \alpha s}|| s|, s\rangle .
$$

This cancels the phase picked up by $\psi$ under the right-multiplication by $e^{\alpha t^{0}}$. Hence, $\tilde{\phi}$ only depends on $p \in O_{m}^{T}$, even though both $\rho^{|s|}(v)$ and $\psi$ depend on $v$.

We now see why this procedure is generally not feasible for UIR's (2.5) labelled by orbits containing spacelike or lightlike momenta, where the stabiliser groups are generated by spacelike and lightlike generators in $\mathfrak{s l}(2, \mathbb{R})$. Under the right-multiplication by $e^{\alpha t^{1}}$ resp. $e^{\alpha t^{+}}$, the elements of (2.5) pick up a phase that cannot be compensated using one of the finite-dimensional irreducible representations of $\operatorname{SL}(2, \mathbb{R})$, as $\rho^{|s|}\left(t^{1}\right)$ has real eigenvalues and $\rho^{|s|}\left(t^{+}\right)$has zero as the sole eigenvalue.

Similar restrictions were found in [10] for the existence of a finite-dimensional covariant description. More general covariant descriptions are given in [18]. However, these are not obtained directly from the standard UIR's of the Poincaré group. Instead, they are constructed using generalised regular representations.

The maps $\tilde{\phi}$ defined in (2.9) satisfy the constraint

$$
\left(i \rho^{|s|}\left(t^{a}\right) p_{a}+m s\right) \tilde{\phi}(p)=0
$$

as can be seen by writing (2.7) as $p_{a} t^{a}=v m t_{0} v^{-1}$ :

$$
\rho^{|s|}\left(t_{a}\right) p_{a} \tilde{\phi}(p)=\rho^{|s|}\left(v m t^{0} v^{-1}\right) \rho^{|s|}(v) \psi(v)|| s|, s\rangle=\psi(v) \rho^{|s|}(v) m i s|| s|, s\rangle=i m s \tilde{\phi}(p),
$$

as required. The equation (2.10) later becomes one of our wave equations and we refer to it as the spin constraint.

Following the method of [31], we now consider extensions of the function $\tilde{\phi}$, defined on the Lie algebra $\mathfrak{s l}(2, \mathbb{R})$. This will enable us to employ a standard Fourier transform for switching from momentum to position space. We embed the timelike orbits $O_{m}^{T}$ into the Lie algebra $\mathfrak{s l}(2, \mathbb{R})$ and define

$$
W_{m s}=\left\{\tilde{\phi}: \mathfrak{s l}(2, \mathbb{R}) \rightarrow \mathbb{C}^{2|s|+1} \mid\left(i \rho^{|s|}\left(t^{a}\right) p_{a}+m s\right) \tilde{\phi}(p)=0,\left(p^{2}-m^{2}\right) \tilde{\phi}(p)=0\right\} .
$$

We call the condition

$$
\left(p^{2}-m^{2}\right) \tilde{\phi}(p)=0
$$

the mass constraint; it is the only condition for spin $s=0$ and we will see that it is implied by the spin constraint for the cases $s= \pm \frac{1}{2}, \pm 1$.

The spaces $W_{m s}$ carry a representation of $\tilde{P}_{3}$ which we shall give below. However, the mass constraint does not fix the sign of $m$. In order to obtain irreducible representations of $\tilde{P}_{3}$, we therefore still need to impose

$$
\Theta\left(-\frac{p_{0}}{m}\right) \tilde{\phi}(p)=0
$$

where $\Theta$ is the Heaviside step function. We call this condition the sign constraint. We remark that though $W_{m s}$ are reducible representations of $\tilde{P}_{3}$, they are irreducible representations of 
a suitable double cover of the extended Poincaré group, which includes time reversal (mapping $O_{m}^{T}$ to $O_{-m}^{T}$ ).

The action of an element $(g, a) \in \tilde{P}_{3}$ on $\tilde{\phi} \in W_{m s}$ is

$$
\left(\pi_{m s}((g, a)) \tilde{\phi}\right)(p)=e^{i a\left(\operatorname{Ad}_{g^{-1}} p\right)} \rho^{|s|}(g) \tilde{\phi}\left(\operatorname{Ad}_{g^{-1}} p\right)
$$

It commutes with the constraints (2.10), (2.11) and (2.12), as required.

Before we can claim that this is an UIR, we need to define the inner product with respect to which the representations are unitary. For spin 0, the invariant inner product on the space $W_{m, s=0}$ is the familiar

$$
\left(\tilde{\phi}_{1}, \tilde{\phi}_{2}\right)=\int_{O_{m}^{T} \cup O_{-m}^{T}} \tilde{\phi}_{1}^{*} \tilde{\phi}_{2} \frac{d p_{1} d p_{2}}{\left|p_{0}\right|},
$$

where the integration is with respect to the standard Lorentz-invariant measure on the mass shell. We will give the inner product for spin $\pm \frac{1}{2}$ and spin \pm 1 below. For a general discussion of the construction of the required invariant scalar product, see [8].

In the case $s=\frac{1}{2}$, the spin constraint (2.10) becomes the Dirac equation in momentum space

$$
\left(i t^{a} p_{a}+\frac{1}{2} m\right) \tilde{\phi}(p)=0
$$

Applying $\left(i t^{a} p_{a}-\frac{1}{2} m\right)$ to this and using (A.4), we see that (2.13) implies the mass constraint (2.11) but not the sign constraint (2.12). However, $\tilde{\phi}$ can be decomposed into positive and negative frequency parts $\tilde{\phi}^{+}$and $\tilde{\phi}^{-}$using a Foldy-Wouthuysen transformation; see [10] for details. This is completely analogous to the situation in $3+1$ dimensions.

To see that (2.13) is indeed the Dirac equation in momentum space, we note that in $2+1$ dimensions, Clifford generators (gamma matrices) satisfying

$$
\left\{\gamma^{a}, \gamma^{b}\right\}=\gamma^{a} \gamma^{b}+\gamma^{b} \gamma^{a}=2 \eta^{a b} \mathrm{id}
$$

can be obtained from the $\mathfrak{s l}(2, \mathbb{R})$ generators $(2.3)$ via

$$
\gamma^{a}=2 i t^{a}
$$

Thus we can write (2.13) as

$$
\left(\gamma^{a} p_{a}+m\right) \tilde{\phi}(p)=0
$$

The invariant scalar product on the space $W_{m, s=\frac{1}{2}}$ is

$$
\left(\tilde{\phi}_{1}, \tilde{\phi}_{2}\right)=\int_{O_{m}^{T} \cup O_{-m}^{T}} \tilde{\phi}_{1}^{\dagger} \gamma^{0} \tilde{\phi}_{2} \frac{d p_{1} d p_{2}}{\left|p_{0}\right|} .
$$

The Lorentz invariance of $\tilde{\phi}_{1}^{\dagger} \gamma^{0} \tilde{\phi}_{2}$ follows from the KAN or Iwasawa decomposition of an element $g \in \mathrm{SL}(2, \mathbb{R})$ into $g=k v$, where $k$ is a rotation (generated by $t^{0}$ and commuting with $\gamma_{0}$ ) and $v$ is of the form

$$
v=\left(\begin{array}{cc}
r & x \\
0 & \frac{1}{r}
\end{array}\right), \quad r>0, \quad x \in \mathbb{R} .
$$

It satisfies $v^{t} \gamma^{0} v=\gamma^{0}$. 
For $s=1, \tilde{\phi}=\tilde{\phi}_{a} t^{a}$ takes values in the adjoint representation of $\mathfrak{s l}(2, \mathbb{R})$. The constraint $(2.10)$ then gives the Proca equations in momentum space

$$
\left(i p_{a} \operatorname{ad}\left(t^{a}\right)+m\right) \tilde{\phi}(p)=0
$$

or

$$
\left[p_{a} t^{a}, \tilde{\phi}(p)\right]=i m \tilde{\phi}(p) .
$$

Taking the Minkowski product (2.4) with $p_{d} t^{d}$ gives

$$
p^{a} \tilde{\phi}_{a}(p)=0 .
$$

The previous two equations together with the identity

$$
[\xi,[\eta, \zeta]]=\left(\xi_{a} \zeta^{a}\right) \eta-\left(\xi_{a} \eta^{a}\right) \zeta, \quad \xi, \eta, \zeta \in \mathfrak{s l}(2, \mathbb{R}), \quad \xi=\xi_{a} t^{a} \quad \text { etc. }
$$

give the mass constraint (2.11). Like for spin $\frac{1}{2}$, the equation (2.10) implies the mass constraint (2.11) but not the sign constraint (2.12).

The invariant scalar product on the space $W_{m, s=1}$ is

$$
\left(\tilde{\phi}_{1}, \tilde{\phi}_{2}\right)=-\int_{O_{m}^{T} \cup O_{-m}^{T}} \tilde{\phi}_{1 a}^{*} \tilde{\phi}_{2}^{a} \frac{d p_{1} d p_{2}}{\left|p_{0}\right|} .
$$

This is manifestly Lorentz invariant, but it may not be obvious that (2.18) is indeed positive definite. This can be seen as follows: due to $(2.17) \tilde{\phi}$ is spacelike, and $\eta$ is negative definite when restricted to spacelike vectors.

The wave equations for the cases $s=-\frac{1}{2}$ and $s=-1$ can be obtained from (2.15) and (2.16) by changing the sign in front of $m$, while the respective inner products stay the same.

\subsection{Fourier transform to position space}

The momentum-space form of the UIR's of $\tilde{P}_{3}$ in the previous sections were designed to be amenable to a standard Fourier transform. Defining

$$
\phi(x)=\int e^{i x(p)} \tilde{\phi}(p) d^{3} p
$$

the spin constraint (2.10) turns into the first order differential equation

$$
\left(\rho^{|s|}\left(t^{a}\right) \partial_{a}+m s\right) \phi(x)=0 .
$$

The mass constraint (2.11) becomes the Klein-Gordon equation

$$
\left(\square+m^{2}\right) \phi=0 .
$$

These are the general wave equations for massive particles with spin $s \in \frac{1}{2} \mathbb{Z}$ in $2+1$ dimensions. An element $(g, a) \in \tilde{P}_{3}$ acts on the wave function $\phi$ via

$$
\left(\pi_{m s}((g, a)) \phi\right)(x)=\rho^{|s|}(g) \phi\left(\operatorname{Ad}_{g}^{*} x+a\right) .
$$

The wave equations for low values of the spin are some of the most studied equations of relativistic physics. For spin 0, the mass constraint is the only constraint, and we obtain the Klein-Gordon equation as already noted above. For spin $\frac{1}{2}$, the spin constraint (2.15) Fourier transforms to the Dirac equation in position space:

$$
\left(i \gamma^{a} \partial_{a}-m\right) \phi=0 \text {. }
$$

For spin 1, the condition (2.16) becomes the Proca equation

$$
\partial_{a}\left[t^{a}, \phi\right]=-m \phi,
$$

and the constraint (2.17) becomes

$$
\partial^{a} \phi_{a}=0 .
$$




\section{Deforming momentum space}

\subsection{The quantum double $\mathcal{D}(\mathrm{SL}(2, \mathbb{R}))$ : motivation and definition}

We now repeat the analysis in the previous section for the case of the quantum double $\mathcal{D}(\mathrm{SL}(2, \mathbb{R}))$ of $\mathrm{SL}(2, \mathbb{R})$, or Lorentz double for short. Before summarising the defining properties of the quantum double of a Lie group, we make a few qualitative remarks which highlight the relation between the Lorentz double and the Poincaré group, following [7, 40].

The action (2.8) of a Poincaré group element on an element of one of its UIR's shows that pure translations act by a multiplication with a special function on the (linear) momentum space $\mathfrak{s l}(2, \mathbb{R})$, namely the plane wave $\psi_{a}(p)=e^{i a(p)}$. In the Lorentz double, this is deformed and generalised: the momentum space is exponentiated and extended to become the whole group manifold $\mathrm{SL}(2, \mathbb{R})$. The space of functions on momentum spaces is generalised to a suitably well-behaved class, for example the class of continuous functions [26]. This deforms the translation part of the Poincaré group into something dual to the rotation/boost part: translations are functions on $\operatorname{SL}(2, \mathbb{R})$ and rotations/boost are elements of $\operatorname{SL}(2, \mathbb{R})$. By allowing linear combinations we obtain a Hopf algebra, consisting of two subalgebras which are in duality.

The quantum double of a Lie group is an example of a quantum double, which in turn is a special class of quantum groups $[13,29]$. It can be defined in various ways. Here we use the form given in $[6,26]$ for locally compact Lie groups. As a vector space, the quantum double $\mathcal{D}(G)$ of a Lie group $G$ is the space of continuous, complex-valued functions $C(G \times G)$. Morally, one should think of this as the tensor product $C(G) \otimes C(G)$, with the first factor being the group algebra and the second factor being the function algebra on $G$. The product in the first factor is by convolution and the product in the second factor is pointwise, but twisted by the action of the first argument. The identity cannot be written as an element of $C(G \times G)$. Strictly speaking it should be added as a separate element, but it is convenient to formally express it as a delta-function.

In the conventions of [31] (which differ from those in $[6,26]$ ), the product $\bullet$, coproduct $\Delta$, unit 1 , co-unit $\varepsilon$, antipode $S$ and $*$-structure are as follows

$$
\begin{aligned}
& \left(F_{1} \bullet F_{2}\right)(g, u):=\int_{G} F_{1}\left(z, z u z^{-1}\right) F_{2}\left(z^{-1} g, u\right) d z, \\
& 1(g, u):=\delta_{e}(g), \\
& (\Delta F)\left(g_{1}, u_{1} ; g_{2}, u_{2}\right):=F\left(g_{1}, u_{1} u_{2}\right) \delta_{g_{1}}\left(g_{2}\right), \\
& \varepsilon(F):=\int_{G} F(z, e) d z \\
& (S F)(g, u):=F\left(g^{-1}, g^{-1} u^{-1} g\right), \\
& F^{*}(g, u):=\overline{F\left(g^{-1}, g^{-1} u g\right)} .
\end{aligned}
$$

In these equations, all integrals over the group are with respect to the Haar measure and $e \in G$ denotes the identity element. The quantum double is quasitriangular [13], and the expression for the $R$-matrix can be found in $[6,26]$. We do not require it here.

\subsection{Coordinates for $\mathrm{SL}(2, \mathbb{R})$}

There are many natural ways to coordinatise the Lie group $\operatorname{SL}(2, \mathbb{R})$, see [4] for a recent review in the context of $2+1$ gravity. Here we use two sets of coordinates, one obtained via the exponential $\operatorname{map} \mathfrak{s l}(2, \mathbb{R}) \rightarrow \mathrm{SL}(2, \mathbb{R})$ and a second which exploits the realisation of $\mathrm{SL}(2, \mathbb{R})$ as a submanifold of $\mathbb{R}^{4}$.

The exponential map exp $: \mathfrak{s l}(2, \mathbb{R}) \rightarrow \mathrm{SL}(2, \mathbb{R})$ is bijective when restricted to a sufficiently small neighbourhood of $0 \in \mathfrak{s l}(2, \mathbb{R})$ and id $\in \mathrm{SL}(2, \mathbb{R})$, but this is not the case globally. In 
fact, it is neither injective nor surjective as we shall see in our discussion of conjugacy classes below. As before, we write elements of $\mathfrak{s l}(2, \mathbb{R})$ as $p=\lambda p_{a} t^{a}$. Using the fact that $\lambda p_{a} t^{a}$ squares to $-\frac{\lambda^{2}}{4} p^{2}$ id, one finds:

$$
\exp \left(\lambda p_{a} t^{a}\right)= \begin{cases}\cos \left(\lambda \sqrt{p^{2}} / 2\right) \mathrm{id}+\frac{p_{a}}{\sqrt{p^{2}} / 2} \sin \left(\lambda \sqrt{p^{2}} / 2\right) t^{a}, & \text { if } p^{2}>0 \\ \mathrm{id}+\lambda p_{a} t^{a}, & \text { if } \quad p^{2}=0 \\ \cosh \left(\lambda \sqrt{-p^{2}} / 2\right) \mathrm{id}+\frac{p_{a}}{\sqrt{-p^{2}} / 2} \sinh \left(\lambda \sqrt{-p^{2}} / 2\right) t^{a}, & \text { if } \quad p^{2}<0 .\end{cases}
$$

It follows from these formulae that elements $u \in \mathrm{SL}(2, \mathbb{R})$ with $\operatorname{tr}(u)<-2$ cannot be written as exponentials. As we shall see in Section 3.3 below, some elements with $\operatorname{tr}(u)=-2$ can also not be written as exponentials. However, we shall also see that if $u$ is not in the image of the exponential map, then $-u$ is. This fact will be useful in Section 4 .

To realise $\mathrm{SL}(2, \mathbb{R})$ as a submanifold of $\mathbb{R}^{4}$, we introduce Cartesian coordinates $\left(\mathcal{P}_{0}, \mathcal{P}_{1}, \mathcal{P}_{2}, \mathcal{P}_{3}\right)$ on $\mathbb{R}^{4}$ and expand

$$
u=\mathcal{P}_{3} \text { id }+\lambda \mathcal{P}_{a} t^{a}=\left(\begin{array}{cc}
\mathcal{P}_{3}+\frac{1}{2} \lambda \mathcal{P}_{1} & \frac{1}{2} \lambda \mathcal{P}_{0}+\frac{1}{2} \lambda \mathcal{P}_{2} \\
-\frac{1}{2} \lambda \mathcal{P}_{0}+\frac{1}{2} \lambda \mathcal{P}_{2} & \mathcal{P}_{3}-\frac{1}{2} \lambda \mathcal{P}_{1}
\end{array}\right),
$$

where Latin indices still take values $0,1,2$. The condition $u \in \operatorname{SL}(2, \mathbb{R})$ is then equivalent to

$$
\operatorname{det} u=\mathcal{P}_{3}^{2}+\frac{\lambda^{2}}{4} \mathcal{P}^{a} \mathcal{P}_{a}=1
$$

We regard $\mathcal{P}_{a}, a=0,1,2$, as the independent coordinates with $\mathcal{P}_{3}= \pm \sqrt{1-\frac{\lambda^{2}}{4} \mathcal{P}^{a} \mathcal{P}_{a}}$. In the following, we refer to the subsets of $\mathrm{SL}(2, \mathbb{R})$ with $\mathcal{P}_{3} \gtrless 0$ as the upper and lower half of $\mathrm{SL}(2, \mathbb{R})$.

Comparing (3.1) and (3.2), we can easily write down a relation between the two coordinate systems on the intersections of their respective patches. The case $p^{2}>0$ is particularly important for us. Here one has

$$
\mathcal{P}_{3}=\cos \left(\lambda \sqrt{p^{2}} / 2\right), \quad \mathcal{P}_{a}=p_{a} \frac{\sin \left(\lambda \sqrt{p^{2}} / 2\right)}{\lambda \sqrt{p^{2}} / 2} .
$$

Taking the limit $\lambda \rightarrow 0$ corresponds to the flattening out of momentum space $\operatorname{SL}(2, \mathbb{R})=$ $\mathrm{AdS}_{3}$. It finally rips apart in the hyperplane of $\mathcal{P}_{3}=0$, producing not one but two copies of flat Minkowski momentum space situated at $\mathcal{P}_{3}= \pm 1$. They would be identified if we had worked with $L_{3}^{+\uparrow}$ instead of $\operatorname{SL}(2, \mathbb{R})$. If, on the other hand, we had worked with the universal covering group, we would have found a countable infinity of copies. For a discussion of $L_{3}^{+\uparrow}$ as momentum space in $(2+1)$-dimensional gravity and $(2+1)$-dimensional non-commutative scalar field theories, see [39].

This property of momentum space is an important consequence of the transition to the double cover or universal cover of $P_{3}$, compounding the more widely known manifestation via the spin of massive particles, which takes integer values in the case of $P_{3}$, half-integer values in the case of $\tilde{P}_{3}$ and real values in the case of the universal cover of $P_{3}$ (see our discussion in Section 2.1).

\subsection{Irreducible representations of $\mathcal{D}(\mathrm{SL}(2, \mathbb{R}))$}

The Lorentz double $\mathcal{D}(\mathrm{SL}(2, \mathbb{R}))$ is a special example of a transformation group algebra, and its UIR's can best be understood in that general context. As shown in [26], they are labelled by conjugacy classes in $\mathrm{SL}(2, \mathbb{R})$ and UIR's of the associated centraliser or stabiliser groups. As 
emphasised in $[7,40]$, this should be seen as a deformation of the picture for the semi-direct product group $\tilde{P}_{3}$. In both cases, the UIR's are labelled by $\operatorname{SL}(2, \mathbb{R})$ orbits in momentum space and UIR's of associated stabilisers. The difference is that momentum space is linear for $\tilde{P}_{3}$ and curved for $\mathcal{D}(\mathrm{SL}(2, \mathbb{R}))$.

The conjugacy classes of $\operatorname{SL}(2, \mathbb{R})$ and their associated stabilisers are classified in [26], and we list them here in a notation adapted to our needs. From the defining property of $\mathrm{SL}(2, \mathbb{R})=$ $\{g \in \mathrm{GL}(2, \mathbb{R}) \mid \operatorname{det}(g)=1\}$ it follows that the (generalised) eigenvalues $\lambda_{1}, \lambda_{2}$ of a given element multiply to one. They are thus either complex conjugate to each other or both real. The set of conjugacy classes can be organised according to the different possible eigenvalues. Some but not all of the conjugacy classes can be obtained from the adjoint orbits in the Lie algebra $\mathfrak{s l}(2, \mathbb{R})$ by exponentiation. We have chosen a labelling of the conjugacy classes which mimicks the conventions we used for the adjoint orbits in the Lie algebra: we use the superscripts T, S and L for 'timelike', 'spacelike' and 'lightlike' to denote conjugacy classes whose elements can be obtained via exponentiated timelike, spacelike or lightlike elements of $\mathfrak{s l}(2, \mathbb{R})$. Our list also includes the stabiliser group of a representative element in each of the conjugacy classes.

T: For $\lambda_{1}=e^{i \frac{\theta}{2}}, \lambda_{2}=e^{-i \frac{\theta}{2}}(0<\theta<2 \pi)$, there are two disjoint families of conjugacy classes, with representative elements $\hat{h}=\exp \left( \pm \theta t^{0}\right)$ which are exponentials of timelike $\mathfrak{s l}(2, \mathbb{R})$ elements. As for the Lie algebra orbits, we introduce a unified notation for the two families, with $\theta \in(0,2 \pi)$ to parametrise one component and $\theta \in(-2 \pi, 0)$ to parametrise the other:

$$
C^{T}(\theta)=\left\{v \exp \left(\theta t^{0}\right) v^{-1} \mid v \in \mathrm{SL}(2, \mathbb{R})\right\}, \quad \theta \in(-2 \pi, 0) \cup(0,2 \pi) .
$$

The stabiliser group is

$$
N^{T}=\left\{\exp \left(\phi t^{0}\right) \mid \phi \in[0,4 \pi)\right\} \simeq \mathrm{U}(1),
$$

with UIR's labelled by $s \in \frac{1}{2} \mathbb{Z}$.

$\mathbf{S}$ : There is one family of conjugacy classes with eigenvalues of the form $\lambda_{1}=e^{\frac{r}{2}}, \lambda_{2}=e^{-\frac{r}{2}}$ $\left(r \in \mathbb{R}_{+}\right)$. Elements of a given conjugacy class are obtained by exponentiating a spacelike Lie algebra element:

$$
C^{S}(r)=\left\{v \exp \left(r t^{1}\right) v^{-1} \mid v \in \mathrm{SL}(2, \mathbb{R})\right\}, \quad r \in \mathbb{R}_{+} .
$$

It has stabiliser group

$$
N^{S}=\left\{ \pm \exp \left(\vartheta t^{1}\right) \mid \vartheta \in \mathbb{R}\right\} \simeq \mathbb{R} \times \mathbb{Z}_{2},
$$

with UIR's labelled by pairs $(b, \epsilon)$, with $b \in \mathbb{R}, \epsilon= \pm 1$.

-S: For $\lambda_{1}=-e^{\frac{r}{2}}, \lambda_{2}=-e^{-\frac{r}{2}}\left(r \in \mathbb{R}_{+}\right)$, there is likewise one family of conjugacy classes which we write as $-C^{S}(r)$. Elements are obtained from those of $C^{S}(r)$ by multiplication with - id; they cannot be written as the exponential of a Lie algebra element. The stabiliser group is again $N^{S}$.

$\mathbf{L}, \mathbf{V}$ : For $\lambda_{1}=\lambda_{2}=1$, we distinguish three conjugacy classes: $C^{V}, C^{L+}$ and $C^{L-}$. The 'vacuum' conjugacy class $C^{V}=\{\mathrm{id}\}$ has stabiliser $\operatorname{SL}(2, \mathbb{R})$, whose UIR's are discussed in [25]. The lightlike conjugacy classes have representative elements $\hat{h}=\exp \left( \pm E_{+}\right)$, which are the exponentials of the lightlike elements $\pm E_{+}$:

$$
C^{L \pm}=\left\{v \exp \left( \pm E_{+}\right) v^{-1} \mid v \in \operatorname{SL}(2, \mathbb{R})\right\} .
$$

The stabiliser group in both cases is

$$
N^{L}=\left\{ \pm \exp \left(z E_{+}\right) \mid z \in \mathbb{R}\right\} \simeq \mathbb{R} \times \mathbb{Z}_{2},
$$

with UIR's labelled by pairs $(b, \epsilon)$, with $b \in \mathbb{R}, \epsilon= \pm 1$. 
-L, - V: For $\lambda_{1}=\lambda_{2}=-1$, we distinguish three conjugacy classes, which are obtained by multiplying $C^{V}, C^{L+}$ and $C^{L-}$ by - id. They have the same stabiliser groups as $C^{V}, C^{L+}$ and $C^{L-}$. Elements of $-C^{L+}$ and $-C^{L-}$ cannot be obtained by exponentiation.

The carrier spaces of the irreducible representations of $\mathcal{D}(\operatorname{SL}(2, \mathbb{R}))$, discussed in [26], are again given in terms of functions on $\mathrm{SL}(2, \mathbb{R})$ satisfying an equivariance condition. The equivariance condition only depends on the stabiliser group of a given conjugacy class, but not directly on the conjugacy class. Since the same stabiliser groups arise for orbits in $\mathfrak{s l}(2, \mathbb{R})$ as for conjugacy classes in $\operatorname{SL}(2, \mathbb{R})$, the general form of the carrier spaces $(2.5)$ of UIR's of $\tilde{P}_{3}$ is unchanged when replacing $\tilde{P}_{3}$ by $\mathcal{D}(\operatorname{SL}(2, \mathbb{R}))$. However, the action of the elements of $\mathcal{D}(\operatorname{SL}(2, \mathbb{R}))$ is different, and does depend on the conjugacy class labelling the representation.

Since we are only able to give covariant forms of momentum constraints in the case of massive particles, i.e., timelike momenta, we restrict ourselves to the corresponding irreducible representations of $\mathcal{D}(\mathrm{SL}(2, \mathbb{R}))$. The relevant conjugacy classes are the conjugacy classes $C^{T}(\theta)$ given in (3.4). Motivated by the application of the Lorentz double to quantum gravity in $2+1$ dimensions, we identify the angle $\theta$ labelling the conjugacy classes with the mass of a particle via $\theta=\lambda m$. This results in a bounded mass, which is a well-known feature of $(2+1)$-dimensional gravity, where $8 \pi G m$ determines a deficit angle in the conical geometry surrounding a particle of mass $m[7,41]$.

Summing up, the irreducible representations of the Lorentz double associated with massive particles are labelled by a mass parameter

$$
m \in\left(-\frac{2 \pi}{\lambda}, 0\right) \cup\left(0, \frac{2 \pi}{\lambda}\right)
$$

and the spin parameter $s \in \frac{1}{2} \mathbb{Z}$. With the carrier space $V_{m s}$ as defined in (2.6), an element $F \in \mathcal{D}(\mathrm{SL}(2, \mathbb{R}))$ acts on $\psi \in V_{m s}$ as

$$
\left(\Pi_{m s}(F) \psi\right)(v)=\int_{\mathrm{SL}(2, \mathbb{R})} F\left(z, z^{-1} v e^{m \lambda t^{0}} v^{-1} z\right) \psi\left(z^{-1} v\right) d z
$$

where we again used the conventions of [31]. In the next section, we adapt the covariantisation procedure of Section 2.3 to this representation.

\subsection{Deformed covariant constraints}

As in Section 2.3, we begin by trading the equivariant function $\psi \in V_{m s}$ for a map

$$
\tilde{\phi}: C^{T}(\lambda m) \rightarrow \mathbb{C}^{2|s|+1}
$$

via

$$
\tilde{\phi}(u)=\psi(v) \rho^{|s|}(v)|| s|, s\rangle,
$$

where the states ||$s|, k\rangle$ are again elements of the basis (A.6) and $u=v e^{m \lambda t^{0}} v^{-1} \in C^{T}(\lambda m)$.

These functions satisfy the analogue of the spin constraint (2.10),

$$
\left(\rho^{|s|}(u)-e^{i \lambda m s}\right) \tilde{\phi}(u)=0
$$

This can be shown by a short calculation which is entirely analogous to that following (2.10). Note that this is a rather natural condition: the value of the function $\tilde{\phi}$ at $u$ lies in the eigenspace of $\rho^{|s|}(u)$ with eigenvalue $e^{i \lambda m s}$. 
We now embed the conjugacy classes $C^{T}(\lambda m)$ into the group $\operatorname{SL}(2, \mathbb{R})$. They are characterised by

$$
\mathcal{P}_{3}=\cos \left(\frac{\lambda m}{2}\right), \quad \frac{\mathcal{P}_{0}}{m}>0 .
$$

In analogy to the conditions (2.11) and (2.12), we refer to the first of these equations as the mass constraint and to the second as the sign constraint. In terms of $u$, the mass constraint is

$$
\left(\frac{1}{2} \operatorname{tr}(u)-\cos \left(\frac{\lambda m}{2}\right)\right) \tilde{\phi}(u)=0 .
$$

We thus define the carrier spaces

$$
\begin{aligned}
\tilde{W}_{m s}= & \left\{\tilde{\phi}: \operatorname{SL}(2, \mathbb{R}) \rightarrow \mathbb{C}^{2|s|+1} \mid\left(\rho^{|s|}(u)-e^{i m \lambda s}\right) \tilde{\phi}(u)=0,\right. \\
& \left.\left(\frac{1}{2} \operatorname{tr}(u)-\cos \left(\frac{\lambda m}{2}\right)\right) \tilde{\phi}(u)=0\right\},
\end{aligned}
$$

and, as in the undeformed case, we will find that the mass constraint is actually implied by the spin constraint for spin $\pm \frac{1}{2}$ and spin \pm 1 . An element $F \in \mathcal{D}(\operatorname{SL}(2, \mathbb{R}))$ acts on $\tilde{\phi} \in \tilde{W}_{m s}$ according to

$$
\left(\Pi_{m s}(F) \tilde{\phi}\right)(u)=\int_{\mathrm{SL}(2, \mathbb{R})} F\left(z, z^{-1} u z\right) \rho^{|s|}(z) \tilde{\phi}\left(z^{-1} u z\right) d z .
$$

For spinless particles, the covariant description involves a function $\tilde{\phi}: \operatorname{SL}(2, \mathbb{R}) \rightarrow \mathbb{C}$. The spin constraint is empty, and we only have the mass constraint (3.7). Writing it in terms of $\mathcal{P}_{3}$ as in (3.6) and applying (3.3), we arrive at

$$
\mathcal{P}_{a} \mathcal{P}^{a} \tilde{\phi}=\left(\frac{\sin (m \lambda / 2)}{\lambda / 2}\right)^{2} \tilde{\phi} .
$$

This is our deformed Klein-Gordon equation in momentum space.

In the case $s=\frac{1}{2}$, we have functions $\tilde{\phi}: \operatorname{SL}(2, \mathbb{R}) \rightarrow \mathbb{C}^{2}$ and the constraint (3.5) becomes simply

$$
u \tilde{\phi}(u)=e^{\frac{i}{2} \lambda m} \tilde{\phi}(u) .
$$

Inserting $u=\mathcal{P}_{3}$ id $+\lambda \mathcal{P}_{a} t^{a}$, this is equivalent to

$$
\lambda \mathcal{P}_{a} t^{a} \tilde{\phi}(u)=\left(e^{\frac{i}{2} \lambda m}-\mathcal{P}_{3}\right) \tilde{\phi}(u) .
$$

However, since the vector $\left(P_{0}, P_{1}, P_{2}\right)$ (like $\left.\left(p_{0}, p_{1}, p_{2}\right)\right)$ is timelike in the case under consideration, the Lie algebra element $\mathcal{P}_{a} t^{a}$ is conjugate to a rotation and has imaginary eigenvalues. Expanding $e^{\frac{i}{2} \lambda m}=\cos (\lambda m / 2)+i \sin (\lambda m / 2)$, the real part of (3.11) is the promised mass constraint $\mathcal{P}_{3} \tilde{\phi}=$ $\cos (\lambda m / 2) \tilde{\phi}$, while the imaginary part is

$$
\left(i \mathcal{P}_{a} t^{a}+\frac{1}{2} \frac{\sin (\lambda m / 2)}{\lambda / 2}\right) \tilde{\phi}(u)=0 .
$$

This is our deformed Dirac equation in momentum space. Using (2.14) to write it in terms of $\gamma$-matrices, we find

$$
\left(\mathcal{P}_{a} \gamma^{a}+\frac{\sin (\lambda m / 2)}{\lambda / 2}\right) \tilde{\phi}(u)=0 .
$$


Applying $\left(\mathcal{P}_{a} \gamma^{a}-\frac{\sin (\lambda m / 2)}{\lambda / 2}\right)$ to (3.13) gives $\mathcal{P}_{a} \mathcal{P}^{a} \tilde{\phi}=\frac{\sin ^{2}(\lambda m / 2)}{\lambda^{2} / 4} \tilde{\phi}$, which is equivalent to the squared version of the mass constraint. Note that the information whether $\tilde{\phi}$ has support on the upper or lower half of $\mathrm{SL}(2, \mathbb{R})$ is not contained in the spin constraint.

For $s=1$, we again work with the adjoint representation of $\operatorname{SL}(2, \mathbb{R})$ and think of $\tilde{\phi}$ as a map $\tilde{\phi}: \operatorname{SL}(2, \mathbb{R}) \rightarrow \mathfrak{s l}(2, \mathbb{R})$, so we can expand $\tilde{\phi}=\tilde{\phi}_{a} t^{a}$. Hence, the constraint (3.5) becomes

$$
u \tilde{\phi}(u) u^{-1}=e^{i \lambda m} \tilde{\phi}(u) .
$$

Expanding again $u=\mathcal{P}_{3}$ id $+\lambda \mathcal{P}_{a} t^{a}$, and using the 'quaternionic' multiplication rule (A.2) of the generators $t^{a}$, we deduce

$$
\lambda \mathcal{P}_{3}\left[\mathcal{P}_{a} t^{a}, \tilde{\phi}\right]-\frac{\lambda^{2}}{2}\left(\mathcal{P}_{a} \mathcal{P}^{a}\right) \tilde{\phi}+\frac{\lambda^{2}}{2}\left(\mathcal{P}^{a} \tilde{\phi}_{a}\right)\left(\mathcal{P}_{b} t^{b}\right)=\left(e^{i \lambda m}-1\right) \tilde{\phi}
$$

where the evaluation at $u$ is understood everywhere. Taking the Minkowski product (2.4) with $\mathcal{P}_{b} t^{b}$ and using that $\left(e^{i \lambda m}-1\right) \neq 0$, we conclude that

$$
\mathcal{P}^{a} \tilde{\phi}_{a}=0 .
$$

Inserting this into (3.14) and applying (3.3) yields

$$
\lambda \mathcal{P}_{3}\left[\mathcal{P}_{a} t^{a}, \tilde{\phi}\right]=\left(e^{i \lambda m}+1-2 \mathcal{P}_{3}^{2}\right) \tilde{\phi}
$$

Again we can argue from the representation theory of $\mathfrak{s l}(2, \mathbb{R})$ reviewed in Appendix A that the eigenvalues of $\left[\mathcal{P}_{a} t^{a}, \cdot\right]$ are imaginary. With $\mathcal{P}_{3}$ real and non-vanishing, we deduce that

$$
\left(\cos (\lambda m)+1-2 \mathcal{P}_{3}^{2}\right) \tilde{\phi}=0,
$$

which is the squared mass constraint

$$
\left(\mathcal{P}_{3}^{2}-\cos ^{2} \frac{\lambda m}{2}\right) \tilde{\phi}=0
$$

Inserting $\mathcal{P}_{3}=\cos \frac{\lambda m}{2}$ into (3.15), we finally arrive at

$$
-i\left[\mathcal{P}_{a} t^{a}, \tilde{\phi}\right]=\frac{\sin (\lambda m / 2)}{\lambda / 2} \tilde{\phi}
$$

This is the deformed Proca equation in momentum space.

The wave equations in momentum space for the cases $s=-\frac{1}{2}$ and $s=-1$ can again be obtained by changing the sign in front of $m$ in (3.13) and (3.16).

\section{Towards non-commutative wave equations}

\subsection{General remarks}

The ordinary Fourier transform, as used in Section 2.4, takes the abelian algebra of functions on a vector space (in our case, momentum space) to the abelian algebra of functions on its dual (in our case, position space). It establishes the link between the UIR's of the Poincaré group and the fundamental wave equations of free, relativistic quantum theory.

Having written some of the irreducible representations of the Lorentz double in terms of $\mathbb{C}^{n}$-valued functions on the deformed momentum space $\mathrm{SL}(2, \mathbb{R})$ obeying Lorentz-covariant constraints, we would now like to use a suitable Fourier transform to obtain wave equations in 
the deformed setting. Our treatment here will be sketchier than in the previous sections, designed to give an overview and to lay the foundation for a future, mathematically more complete treatment. We consider two kinds of Fourier transform.

One version, called quantum group Fourier transform in the following, takes elements of a given Hopf algebra to elements of its dual Hopf algebra [24, 29]; it is defined in a rather general Hopf-algebraic setting and can, in particular, be applied to the Hopf algebra of functions on a Lie group.

A second version maps functions on a Lie group $G$ to functions on the dual of the Lie algebra $\mathfrak{g}^{*}$, equipped with a $\star$-product. This is studied in different guises in $[14,16,17,23,38]$ for the case of $G$ being the rotation group in three dimensions (or its cover). It is investigated in a more general setting of Lie groups satisfying certain technical requirements in $[20,36]$. We call it group Fourier transform in the following ${ }^{2}$. The paper [17] also includes a discussion of the relation between these two kinds of Fourier transforms.

\subsection{Quantum group Fourier transform}

In our deformed theory, momentum space is $\operatorname{SL}(2, \mathbb{R})$ and the 'algebra of momenta' is the algebra $C(\mathrm{SL}(2, \mathbb{R}))$ of (suitably well-behaved) functions on $\mathrm{SL}(2, \mathbb{R})$, with pointwise multiplication. This is a commutative but not co-commutative algebra. The quantum group Fourier transform maps elements of this algebra to elements of the dual 'position algebra', which can be taken to be a suitable class of functions on $\mathrm{SL}(2, \mathbb{R})$ with multiplication given by convolution (i.e., a suitable version of the group algebra) or the universal enveloping algebra $\mathrm{U}(\mathfrak{s l}(2, \mathbb{R}))$, with generators

$$
\hat{x}^{a}=i \lambda t^{a}
$$

satisfying the $\mathfrak{s l}(2, \mathbb{R})$ commutation relations

$$
\left[\hat{x}^{a}, \hat{x}^{b}\right]=i \lambda \epsilon^{a b c} \hat{x}_{c} \text {. }
$$

Note that this non-commutative 'spin spacetime' has a long history in the literature of $(2+1)$ dimensional quantum gravity, see for example the papers [15, 32, 44]. It is naturally accommodated in the framework of the Lorentz double, for which, in the terminology of [9], $\mathrm{U}(\mathfrak{s l}(2, \mathbb{R}))$ is the 'Schrödinger representation'.

In [9], the authors consider the Euclidean situation $\mathrm{U}(\mathfrak{s u}(2))$, and go on to develop a bicovariant calculus on $\mathrm{U}(\mathfrak{s u}(2))$ and to study the quantum group Fourier transform in this case. This was used in [31] to derive non-commutative linear differential equations characterising irreducible representations of the double $\mathcal{D}(\mathrm{SU}(2))$. We will now show how most of these results can be adapted, at least formally, to the Lorentzian setting.

The required quantum group Fourier transform is a map from a suitable class of functions $C(\mathrm{SL}(2, \mathbb{R}))$ to a suitable closure of $\mathrm{U}(\mathfrak{s l}(2, \mathbb{R}))$. This closure should include group elements $u \in \mathrm{SL}(2, \mathbb{R})$, viewed as infinite power series in $\mathrm{U}(\mathfrak{s l}(2, \mathbb{R}))$. The fact that the exponential map is not surjective for $\operatorname{SL}(2, \mathbb{R})$ does not pose any difficulties here since all elements of $\operatorname{SL}(2, \mathbb{R})$ can be written as $\pm \exp (\xi)$ for some $\xi \in \mathfrak{s l}(2, \mathbb{R})$, see our classification of conjugacy classes in Section 3.3. In order to accommodate the $\mathbb{C}^{n}$-valued functions in the carrier space (3.8), we tensor both $C(\mathrm{SL}(2, \mathbb{R}))$ and $\mathrm{U}(\mathfrak{s l}(2, \mathbb{R}))$ with $\mathbb{C}^{n}$.

The 'plane waves' used in this quantum group Fourier transforms are simply the group elements of $\operatorname{SL}(2, \mathbb{R})$, viewed as functions of the non-commutative position vector $\hat{x}=\left(\hat{x}^{0}, \hat{x}^{1}, \hat{x}^{2}\right)$ according to

$$
\psi(u ; \hat{x}):=u= \pm \exp \left(-i p_{a} \hat{x}^{a}\right)= \pm \exp \left(\lambda p_{a} t^{a}\right) \in \mathrm{SL}(2, \mathbb{R}) .
$$

\footnotetext{
${ }^{2}$ The name 'non-commutative Fourier transform' is also frequently used in the literature, but since noncommutativity is also a feature of the quantum group Fourier transform we prefer the name 'group Fourier transform' here.
} 
The quantum group Fourier transform of

$$
\tilde{\phi}: \operatorname{SL}(2, \mathbb{R}) \rightarrow \mathbb{C}^{n}
$$

is then

$$
\phi(\hat{x})=\int_{\mathrm{SL}(2, \mathbb{R})} d u \psi(u ; \hat{x}) \tilde{\phi}(u)
$$

where $d u$ is the Haar measure on $\operatorname{SL}(2, \mathbb{R})$. The expression (4.2) is formal and the analogue of the corresponding expressions for the Euclidean version used in [17]. Even in that context, it has not been defined in a mathematically rigorous fashion.

Adapting the bi-covariant calculus developed in [9] to the Lorentzian 'spin spacetime' $\mathrm{U}(\mathfrak{s l}(2, \mathbb{R}))$ requires a vector space on which $\mathrm{U}(\mathfrak{s l}(2, \mathbb{R}))$ acts from both the left and the right (i.e., a bimodule). As for $\mathrm{U}(\mathfrak{s u}(2))$, we can use the space $M_{2}(\mathbb{C})$ of complex $2 \times 2$ matrices on which the generators $t_{a}$ of $\mathrm{U}(\mathfrak{s l}(2, \mathbb{R}))$ act via left- and right-multiplication in the fundamental representation (A.1). Differential 1-forms are elements of $M_{2}(\mathbb{C}) \otimes \mathrm{U}(\mathfrak{s l}(2, \mathbb{R}))$, and the Lorentzian version of the four-dimensional calculus developed in [9] gives the exterior derivative of group-like elements as

$$
\mathrm{d} u=\frac{1}{\lambda}(u-\mathrm{id}) \otimes u
$$

where id is the $2 \times 2$ identity matrix. Partial derivatives can be computed by expanding the right-hand side in the basis

$$
e_{3}=\mathrm{id}, \quad e_{a}=-i t_{a}, \quad a=0,1,2 .
$$

In our coordinates (3.2), we have $u=\mathcal{P}_{3}$ id $+\lambda \mathcal{P}_{a} t^{a}$ and find

$$
\partial_{3} u=\frac{1}{\lambda}\left(\mathcal{P}_{3}-1\right) u, \quad \partial_{a} u=i \mathcal{P}_{a} u, \quad a=0,1,2 .
$$

Assuming the validity of the Fourier transform (4.2), non-commutative wave equations can now easily be obtained from our momentum constraints in Section 3.4. The constraint (3.9) implies the non-commutative Klein-Gordon equation

$$
\left(\partial_{a} \partial^{a}+\left(\frac{\sin (m \lambda / 2)}{\lambda / 2}\right)^{2}\right) \phi=0 .
$$

The deformed spin $\frac{1}{2}$ constraint (3.13) takes the from of a non-commutative Dirac equation

$$
\left(i \partial_{a} \gamma^{a}-\frac{\sin (\lambda m / 2)}{\lambda / 2}\right) \phi=0
$$

and the deformed Proca constraint (3.16) turns into the non-commutative Proca equation

$$
\partial_{a}\left[t^{a}, \phi\right]=-\frac{\sin (\lambda m / 2)}{\lambda / 2} \phi
$$

which implies $\partial^{a} \phi_{a}=0$. 


\subsection{Group Fourier transform}

We turn to the group Fourier transform of functions of the form (4.1). This time, the image of the Fourier transform is a certain class of function on ordinary $\mathbb{R}^{3}$, equipped with a $\star$-product. As in our discussion of the quantum group Fourier transform, we will sketch the main ideas here, leaving a careful treatment for future work. Our main references are [14, 16, 17, 23, 38] which deal with the case of $\mathrm{SU}(2)$ and $\mathrm{SO}(3)$, and [20,36] for a more general discussion of the group Fourier transform. These papers discuss different possibilities of implementing the group Fourier transform and the associated $\star$-products. The starting point for each possibility is a choice of plane wave, which, for a general Lie group $G$, is a map

$$
\psi_{\star}: G \times \mathfrak{g}^{*} \rightarrow \mathbb{C},
$$

satisfying a completeness condition [36]

$$
\int_{\mathfrak{g}^{*}} d x \psi(u, x)=\delta_{e}(u),
$$

where $d x$ is a (suitably normalised) measure on the vector space $\mathfrak{g}^{*}$, and $\delta_{e}$ is the Dirac delta distribution at the identity element $e \in G$. Evaluating the plane wave on a given $u \in G$ produces functions on $\mathfrak{g}^{*}$ for which we define a $\star$-product via

$$
\psi_{\star}\left(u^{(1)}, x\right) \star \psi_{\star}\left(u^{(2)}, x\right)=\psi_{\star}\left(u^{(1)} u^{(2)}, x\right) .
$$

This induces a $\star$-product on the space $L_{\star}^{2}\left(\mathfrak{g}^{*}\right)$ of all functions on $\mathfrak{g}^{*}$ which can be written as the group Fourier transform of some $\tilde{\phi} \in C(G)$ :

$$
\phi(x)=\int_{G} d u \psi_{\star}(u, x) \tilde{\phi}(u) .
$$

Even for the most studied case $G=\mathrm{SU}(2), \mathfrak{g}^{*} \simeq \mathbb{R}^{3}$, it is not easy to write down a suitable plane wave. Several options have been considered in the literature, each with its own advantages and drawbacks.

In [16], the authors study a plane wave which is defined for the quotient $\mathrm{SO}(3) \simeq \mathrm{SU}(2) / \mathbb{Z}_{2}$. This is reviewed in [17], where the authors then go on to treat the case $G=\mathrm{SU}(2)$ by extending it centrally to $\mathbb{R}^{+} \times \mathrm{SU}(2)$. In [14], plane waves for $G=\mathrm{SU}(2)$ are constructed by using a spinorial parametrisation of $\mathbb{R}^{3}$ (essentially by using $x \in \mathbb{R}^{3}$ to parametrise a projection operator onto $\mathrm{SU}(2)$ eigenstates) while in [20] plane waves for $\mathrm{SU}(2)$ are constructed using a parametrisation via the exponential map.

All these constructions can be adapted with different degrees of completeness to the case at hand, i.e., $G=\operatorname{SL}(2, \mathbb{R}), \mathfrak{g}^{*} \simeq \mathbb{R}^{3}$. One way to bring out the similarities is to view $\mathrm{SU}(2)$ as the group of unit quaternions and $\operatorname{SL}(2, \mathbb{R})$ as the group of unit pseudo-quaternions, see [35] for a detailed discussion of this point of view in the context of $2+1$ gravity. The parametrisation $(3.2)$ of $u \in \mathrm{SL}(2, \mathbb{R})$ is essentially a quaternionic parametrisation, with $\lambda t_{a}$ playing the role of imaginary pseudo-quaternions and id being the identity in the pseudo-quaternions. Then the central extensions $\mathbb{R}^{+} \times \mathrm{SU}(2)$ and $\mathbb{R}^{+} \times \mathrm{SL}(2, \mathbb{R})$ are simply the groups of all quaternions and, respectively, pseudo-quaternions.

For the purposes of our overview and outlook, we will illustrate these general remarks by considering two plane waves and using them to Fourier transform the momentum space constraints of Section 3.4.

The first is defined on $L_{3}^{+\uparrow} \simeq \operatorname{SL}(2, \mathbb{R}) / \mathbb{Z}_{2}$ and is the analogue of the 'bosonic' plane wave defined on $\mathrm{SO}(3)[16,17]$. It is the map

$$
\psi_{\star}^{B}: \operatorname{SL}(2, \mathbb{R}) / \mathbb{Z}_{2} \times \mathbb{R}^{3} \rightarrow \mathbb{C}, \quad \psi_{\star}^{B}(u, x)=\exp \left(i \epsilon\left(\mathcal{P}_{3}\right) \mathcal{P}^{a} x_{a}\right),
$$


where $u \in \mathrm{SL}(2, \mathbb{R})$ is again parametrised as in $(3.2), \epsilon\left(\mathcal{P}_{3}\right)$ is the sign of $\mathcal{P}_{3}$, and $x \in \mathfrak{s l}(2, \mathbb{R})^{*} \simeq$ $\mathbb{R}^{3}$ as in Section 2. The inclusion of the sign of $\mathcal{P}_{3}$ means that the argument of the exponential is invariant under the $\mathbb{Z}_{2}$ quotient $u \mapsto-u$ and therefore a function on the quotient $\operatorname{SL}(2, \mathbb{R}) / \mathbb{Z}_{2}$. As in the Euclidean case, it is not defined on the set of measure zero where $\mathcal{P}_{3}=0$.

The multiplication via the $\star$-product (4.6) implies

$$
\exp \left(i \epsilon\left(\mathcal{P}_{3}^{(1)}\right) \mathcal{P}_{a}^{(1)} x^{a}\right) \star \exp \left(i \epsilon\left(\mathcal{P}_{3}^{(2)}\right) \mathcal{P}_{a}^{(2)} x^{a}\right)=\exp \left(i \epsilon\left(\mathcal{P}_{3}^{(1 \oplus 2)}\right) \mathcal{P}_{a}^{(1 \oplus 2)} x^{a}\right),
$$

with $\mathcal{P}_{3}^{(1 \oplus 2)}$ and $\mathcal{P}_{a}^{(1 \oplus 2)}, a=0,1,2$, defined via

$$
u^{(1)} u^{(2)}=\mathcal{P}_{3}^{(1 \oplus 2)} \mathrm{id}+\lambda \mathcal{P}_{a}^{(1 \oplus 2)} t_{a} .
$$

Turning now to the covariant momentum space constraints defining irreducible representations of $\mathcal{D}(\mathrm{SL}(2, \mathbb{R}))$, we note that momentum space constraints for the bosonic fields (3.9) and (3.16) make sense for functions $\tilde{\phi}$ on $\operatorname{SL}(2, \mathbb{R})$ which are invariant under $u \rightarrow-u$ and thus defined on $\operatorname{SL}(2, \mathbb{R}) / \mathbb{Z}_{2}$, while the spin $\frac{1}{2}$ constraint (3.12) does not descend to the quotient. The group Fourier transform

$$
\phi_{\star}^{B}(x)=\int_{\mathrm{SL}(2, \mathbb{R}) / \mathbb{Z}_{2}} d u \psi_{\star}^{B}(u, x) \tilde{\phi}(u)
$$

turns the momentum space constraints for the bosonic fields (3.9) and (3.16) into formally the same equations as (4.3) and (4.5) for $\phi_{\star}^{B}(x)$, but with $\partial_{a}$ now denoting the usual partial derivative $\partial / \partial x^{a}$.

The second form of plane wave we want to consider here uses the exponential map to parametrise $\mathrm{SL}(2, \mathbb{R})$. Although the exponential map $\mathfrak{s l}(2, \mathbb{R}) \rightarrow \mathrm{SL}(2, \mathbb{R})$ is not surjective and therefore cannot be used to parametrise the entire group, its image includes the 'inside of the lightcone'

$$
\mathrm{SL}(2, \mathbb{R})_{ \pm}=\bigcup_{\lambda m \in(-2 \pi, 0) \cup(0,2 \pi)} C^{T}(\lambda m)
$$

on which the elements of massive irreducible representations have their support. For the purpose of Fourier transforming the momentum space constraints of Section 3.4, it is therefore sufficient to consider elements in $\mathrm{SL}(2, \mathbb{R})_{ \pm}$for which we can define the 'exponential' plane wave

$$
\psi_{\star}^{E}(u, x)=\exp \left(i x^{a} p_{a}\right), \quad u=\exp \left(\lambda p_{a} t^{a}\right) \in \mathrm{SL}(2, \mathbb{R})_{ \pm} .
$$

The associated group Fourier transform maps this to

$$
\phi_{\star}^{E}(x)=\int_{\mathrm{SL}(2, \mathbb{R})_{ \pm}} d u \psi_{\star}^{E}(u, x) \tilde{\phi}(u) .
$$

The momentum constraints (3.9), (3.12) and (3.16) on $\tilde{\phi}$ imply equations for $\phi_{\star}^{E}(x)$ involving exponentiated differential operators.

For spin $\frac{1}{2}$, the left hand side of (3.10) produces the exponentiated Dirac operator

$$
e^{-\frac{\lambda}{2} \gamma^{a} \partial_{a}} \phi_{\star}^{E}(x),
$$

which was considered in a very different context by Atiyah and Moore in [5]. The authors considered difference-differential versions of several fundamental equations of physics, including the Dirac equation, allowing for advanced and retarded as well as advanced-retarded versions. For spin $\frac{1}{2}$, this involves in an essential way the exponential of the Dirac operator. Their work 
stresses the relation between exponentiated differential operators and difference equations, and explores the consequences of using such equations in fundamental physics.

The appearance of difference-differential equations in theories with curved momentum space was also pointed out in [32] in the context of $(2+1)$-dimensional gravity. However, with few exceptions [37], this point of view has not received much attention in the context of generalised Fourier transforms and quantum groups.

\section{Conclusion and outlook}

In this paper, we studied the consequences of curved momentum space for the spacetime physics of massive and spinning particles in a particular model. We have kept an open mind about the motivation for studying curved momentum space. The model considered here comes from $(2+1)$-dimensional quantum gravity, and has the added benefit of maintaing (a deformed version of) Poincaré symmetry. However, as reviewed in the Introduction, curved momentum space arises in several contexts and has been of theoretical interest at least since Born's pioneering considerations in [11].

Our interest in the spacetime description of the particles via relativistic wave equations stems from the fundamental role such equations play in relativistic quantum mechanics and quantum field theory. The wave equations, not the equivalent momentum constraints, provide the standard route for constructing interacting theories, be it via coupling to an external classical field (as in the Dirac equation for the electron in the hydrogen atom) or in a fully interacting quantum field theory. It is therefore interesting to see how relativistic wave equations are modified when momentum space becomes curved.

In Section 4, we gave an overview over different ways of obtaining the wave equations from the covariant momentum constraints derived earlier via Fourier transforms. Our discussion there should be viewed as a first step, pulling together relevant approaches in the literature and preparing the ground for a mathematically precise treatment. At this stage, our findings can be summarised as follows.

The quantum group Fourier transform is perhaps the most elegant method of Fourier transforming the covariant momentum space constraints, and directly takes us into the realm of non-commutative geometry. However, this route is strewn with considerable mathematical challenges. At present, the formula (4.2) leads to equations like (4.4) which are re-interpretations of mathematically well-defined momentum-space constraints but do not have an independent mathematically rigorous definition.

The group Fourier transform, by contrast, leads to equations on ordinary $\mathbb{R}^{3}$ which have an independent mathematical meaning as differential or difference-differential equations. However, here the nature of the equation depends on the choice of plane wave. We have only considered two choices of plane waves here, and found conventional wave equations from the 'bosonic' plane wave (4.7) but difference-differential equations from the 'exponential' plane wave (4.8). The latter are suggestive of a discrete spacetime geometry as considered in [32] and are interesting from the more general viewpoint emphasised in [5].

There are several avenues for developing the work started here. One would like to extend the set of wave equations to include equations for massless particles and for particles with spins other than $0, \frac{1}{2}$ and 1 . Anyonic excitations are relevant and interesting in this context. They arise naturally in the context of $2+1$ gravity, where the spin is quantised in units which depend on the mass [7]. To study them one needs to work with the universal cover of $\operatorname{SL}(2, \mathbb{R})$.

It would also be interesting to consider both the quaternionic extension $\mathbb{R}^{+} \times \operatorname{SL}(2, \mathbb{R})$ and a Lorentzian version of the spinorial description in [14] in future work. This will presumably lead to yet different types of 'wave equations'. 
On general grounds, the choice of coordinates on the group $\operatorname{SL}(2, \mathbb{R})$ should not matter, and one expects plane waves which differ only by different coordinates to lead to equivalent wave equations after Fourier transform. However, in so far as different plane waves reflect a different choice of group (e.g. $\mathrm{SL}(2, \mathbb{R}) / \mathbb{Z}_{2}$ versus $\mathrm{SL}(2, \mathbb{R})$ or $\mathbb{R}^{+} \times \mathrm{SL}(2, \mathbb{R})$ ), one would expect the associated wave equations to be different.

Ultimately, one would like to achieve a systematic understanding of the nature and interrelationship of wave equations that can be obtained via Fourier transform of a given curved momentum space constraint.

\section{A Basis and finite-dimensional representations of $\mathfrak{s l}(2, \mathbb{R})$}

In the main text, we use the the basis $\left\{t^{a}, a=0,1,2\right\}$ of $\mathfrak{s l}(2, \mathbb{R})$ with

$$
t^{0}=\frac{1}{2}\left(\begin{array}{rr}
0 & 1 \\
-1 & 0
\end{array}\right), \quad t^{1}=\frac{1}{2}\left(\begin{array}{rr}
1 & 0 \\
0 & -1
\end{array}\right), \quad t^{2}=\frac{1}{2}\left(\begin{array}{ll}
0 & 1 \\
1 & 0
\end{array}\right) .
$$

The basis elements satisfy

$$
t^{a} t^{b}=-\frac{1}{4} \eta^{a b} \mathrm{id}+\frac{1}{2} \epsilon^{a b c} t_{c}
$$

where id denotes the $2 \times 2$ identity matrix. As a result, we have the commutation relations

$$
\left[t^{a}, t^{b}\right]=\epsilon^{a b c} t_{c}
$$

and the anticommutation relations

$$
\left\{t^{a}, t^{b}\right\}=t^{a} t^{b}+t^{b} t^{a}=-\frac{1}{2} \eta^{a b} \mathrm{id} .
$$

Finally, we note the orthogonality relations

$$
-2 \operatorname{tr}\left(t^{a} t^{b}\right)=\eta^{a b}
$$

The representation theory of $\mathfrak{s l}(2, \mathbb{R})$ is best studied in terms of raising and lowering operators

$$
H=t^{1}, \quad E_{+}=t^{2}+t^{0}=\left(\begin{array}{cc}
0 & 1 \\
0 & 0
\end{array}\right), \quad E_{-}=t^{2}-t^{0}=\left(\begin{array}{ll}
0 & 0 \\
1 & 0
\end{array}\right),
$$

with commutation relations

$$
\left[H, E_{+}\right]=E_{+}, \quad\left[H, E_{-}\right]=-E_{-}, \quad\left[E_{+}, E_{-}\right]=2 H .
$$

It is well-known [43] that the finite-dimensional representations of the Lie algebra $\mathfrak{s l}(2, \mathbb{R})$ are parametrised by $j \in \frac{1}{2}(\mathbb{N} \cup 0)$. For each value of $j$ there is a unique irreducible representation $\rho^{j}$ on $V_{j} \simeq \mathbb{C}^{2 j+1}$. The standard basis $\left\{w_{j}, w_{j-1}, \ldots, w_{1-j}, w_{-j}\right\}$ of $V_{j}$ is such that

$$
\rho^{j}(H) w_{k}=k w_{k}, \quad \rho^{j}\left(E_{-}\right) w_{k}=(j+k) w_{k-1}, \quad \rho^{j}\left(E_{+}\right) w_{k}=(j-k) w_{k+1} .
$$

These representations are not unitary. Only $\rho^{j}\left(t^{0}\right)$ has imaginary eigenvalues and exponentiates to a unitary matrix. In the main text, we work with an eigenbasis

$$
\{|j, j\rangle,|j, j-1\rangle, \ldots,|j, 1-j\rangle,|j,-j\rangle\}
$$

of $\rho^{j}\left(t^{0}\right)$ satisfying $\rho^{j}\left(t^{0}\right)|j, k\rangle=i k|j, k\rangle$. 


\section{Acknowledgements}

MW thanks the Department of Mathematics at Heriot-Watt University for hospitality during a six-months visit in 2010 when the bulk of the research reported here was carried out. BJS thanks Sergio Inglima for discussions and comments on a draft version of the manuscript. Both MW and BJS thank the University of Ghana for hospitality during a research visit in April 2010 .

\section{References}

[1] Achúcarro A., Townsend P.K., A Chern-Simons action for three-dimensional anti-de Sitter supergravity theories, Phys. Lett. B 180 (1986), 89-92.

[2] Amelino-Camelia G., Doubly-special relativity: facts, myths and some key open issues, Symmetry 2 (2010), 230-271, arXiv:1003.3942.

[3] Amelino-Camelia G., Freidel L., Kowalski-Glikman J., Smolin L., The principle of relative locality, Phys. Rev. D 84 (2011), 084010, 13 pages, arXiv:1101.0931.

[4] Arzano M., Latini D., Lotito M., Group momentum space and Hopf algebra symmetries of point particles coupled to $2+1$ gravity, arXiv:1403.3038.

[5] Atiyah M.F., Moore G.W., A shifted view of fundamental physics, arXiv:1009.3176.

[6] Bais F.A., Muller N.M., Topological field theory and the quantum double of SU(2), Nuclear Phys. B 530 (1998), 349-400, hep-th/9804130.

[7] Bais F.A., Muller N.M., Schroers B.J., Quantum group symmetry and particle scattering in (2+1)dimensional quantum gravity, Nuclear Phys. B 640 (2002), 3-45, hep-th/0205021.

[8] Barut A.O., Ra̧czka R., Theory of group representations and applications, 2nd ed., World Scientific Publishing Co., Singapore, 1986.

[9] Batista E., Majid S., Noncommutative geometry of angular momentum space U(su(2)), J. Math. Phys. 44 (2003), 107-137, hep-th/0205128.

[10] Binegar B., Relativistic field theories in three dimensions, J. Math. Phys. 23 (1982), 1511-1517.

[11] Born M., A suggestion for unifying quantum theory and relativity, Proc. R. Soc. Lond. Ser. A 165 (1938), 291-303.

[12] de Sousa Gerbert P., On spin and (quantum) gravity in 2+1 dimensions, Nuclear Phys. B 346 (1990), $440-472$.

[13] Drinfel'd V.G., Quantum groups, in Proceedings of the International Congress of Mathematicians, Vol. 1, 2 (Berkeley, Calif., 1986), Amer. Math. Soc., Providence, RI, 1987, 798-820.

[14] Dupuis M., Girelli F., Livine E., Spinors and Voros star-product for group field theory: first contact, Phys. Rev. D 86 (2012), 105034, 5 pages, arXiv:1107.5693.

[15] Freidel L., Livine E.R., 3D quantum gravity and effective noncommutative quantum field theory, Phys. Rev. Lett. 96 (2006), 221301, 4 pages, hep-th/0512113.

[16] Freidel L., Livine E.R., Ponzano-Regge model revisited. III. Feynman diagrams and effective field theory, Classical Quantum Gravity 23 (2006), 2021-2061, hep-th/0502106.

[17] Freidel L., Majid S., Noncommutative harmonic analysis, sampling theory and the Duflo map in $2+1$ quantum gravity, Classical Quantum Gravity 25 (2008), 045006, 37 pages, hep-th/0601004.

[18] Gitman D.M., Shelepin A.L., Poincaré group and relativistic wave equations in $2+1$ dimensions, J. Phys. A: Math. Gen. 30 (1997), 6093-6121.

[19] Grigore D.R., The projective unitary irreducible representations of the Poincaré group in $1+2$ dimensions, J. Math. Phys. 34 (1993), 4172-4189, hep-th/9304142.

[20] Guedes C., Oriti D., Raasakka M., Quantization maps, algebra representation, and non-commutative Fourier transform for Lie groups, J. Math. Phys. 54 (2013), 083508, 31 pages, arXiv:1301.7750.

[21] Imai S., Sasakura N., Scalar field theories in a Lorentz-invariant three-dimensional noncommutative spacetime, J. High Energy Phys. 2000 (2000), no. 9, 032, 23 pages, hep-th/0005178.

[22] Jackiw R., Nair V.P., Relativistic wave equation for anyons, Phys. Rev. D 43 (1991), 1933-1942. 
[23] Joung E., Mourad J., Noui K., Three dimensional quantum geometry and deformed symmetry, J. Math. Phys. 50 (2009), 052503, 29 pages, arXiv:0806.4121.

[24] Kempf A., Majid S., Algebraic q-integration and Fourier theory on quantum and braided spaces, J. Math. Phys. 35 (1994), 6802-6837, hep-th/9402037.

[25] Knapp A.W., Representation theory of semisimple groups. An overview based on examples, Princeton Mathematical Series, Vol. 36, Princeton University Press, Princeton, NJ, 1986.

[26] Koornwinder T.H., Muller N.M., The quantum double of a (locally) compact group, J. Lie Theory 7 (1997), 101-120, q-alg/9712042.

[27] Lukierski J., Ruegg H., Nowicki A., Tolstoy V.N., q-deformation of Poincaré algebra, Phys. Lett. B 264 (1991), 331-338.

[28] Majid S., Noncommutative-geometric groups by a bicrossproduct construction: Hopf algebras at the Planck scale, Ph.D. Thesis, Harvard University, 1988.

[29] Majid S., Foundations of quantum group theory, Cambridge University Press, Cambridge, 1995.

[30] Majid S., Ruegg H., Bicrossproduct structure of $\kappa$-Poincaré group and non-commutative geometry, Phys. Lett. B 334 (1994), 348-354, hep-th/9405107.

[31] Majid S., Schroers B.J., q-deformation and semidualization in 3D quantum gravity, J. Phys. A: Math. Theor. 42 (2009), 425402, 40 pages, arXiv:0806.2587.

[32] Matschull H.J., Welling M., Quantum mechanics of a point particle in (2+1)-dimensional gravity, Classical Quantum Gravity 15 (1998), 2981-3030, gr-qc/9708054.

[33] Meusburger C., Schroers B.J., Poisson structure and symmetry in the Chern-Simons formulation of (2+1)dimensional gravity, Classical Quantum Gravity 20 (2003), 2193-2233, gr-qc/0301108.

[34] Meusburger C., Schroers B.J., The quantisation of Poisson structures arising in Chern-Simons theory with gauge group $G \ltimes \mathfrak{g}^{*}, A d v$. Theor. Math. Phys. 7 (2003), 1003-1043, hep-th/0310218.

[35] Meusburger C., Schroers B.J., Quaternionic and Poisson-Lie structures in three-dimensional gravity: the cosmological constant as deformation parameter, J. Math. Phys. 49 (2008), 083510, 27 pages, arXiv:0708.1507.

[36] Raasakka M., Group Fourier transform and the phase space path integral for finite dimensional Lie groups, arXiv:1111.6481.

[37] Sasai Y., Sasakura N., Domain wall solitons and Hopf algebraic translational symmetries in noncommutative field theories, Internat. J. Modern Phys. A 23 (2008), 2277-2278, arXiv:0711.3059.

[38] Sasai Y., Sasakura N., The Cutkosky rule of three dimensional noncommutative field theory in Lie algebraic noncommutative spacetime, J. High Energy Phys. 2009 (2009), no. 6, 013, 22 pages, arXiv:0902.3050.

[39] Sasai Y., Sasakura N., Massive particles coupled with 2+1 dimensional gravity and noncommutative field theory, arXiv:0902.3502.

[40] Schroers B.J., Combinatorial quantisation of Euclidean gravity in three dimensions, in Quantization of Singular Symplectic Quotients, Progress in Mathematics, Vol. 198, Editors N.P. Landsman, M. Pflaum, M. Schlichenmaier, Birkhäuser Verlag, Basel, 2001, 307-328, math.QA/0006228.

[41] Schroers B.J., Quantum gravity and non-commutative spacetimes in three dimensions: a unified approach, Acta Phys. Polon. B Proc. Suppl. 4 (2011), 379-402, arXiv:1105.3945.

[42] Snyder H.S., Quantized space-time, Phys. Rev. 71 (1947), 38-41.

[43] Sternberg S., Group theory and physics, Cambridge University Press, Cambridge, 1994.

[44] 't Hooft G., Quantization of point particles in (2+1)-dimensional gravity and spacetime discreteness, Classical Quantum Gravity 13 (1996), 1023-1039, gr-qc/9601014.

[45] Witten E., 2+1-dimensional gravity as an exactly soluble system, Nuclear Phys. B 311 (1988), 46-78. 